\title{
The interface of obesity and depression: risk factors for the metabolic syndrome
}

Obesity, defined as excess body fat, ${ }^{1}$ is a multigenic behavioral disorder resulting from gene-environment interactions. Body weight is determined by two behaviors: food intake and energy expenditure. While considerable attention is paid to the metabolic and cardiovascular consequences of obesity, its behavioral determinants are not sufficiently addressed by current research. In recent years there has been increased attention paid to the central circuitry regulating food intake and body weight. ${ }^{2}$ Those studies show a considerable overlap between the candidate systems involved in obesity and those involved in depression. We would like to make the case here that obesity and depression have considerable overlap at multiple levels from the pathophysiologic, to the metabolic, and therapeutic (Table).

While humans are well adapted to starvation as the result of thousands of years of exposure to cycles of feast and famine, they are poorly adapted to overnutrition. ${ }^{3}$ In fact, humans have only had a continuous surplus of food for the past 100 years. Therefore, it is not surprising that there has not been a genetic adaptation to overnutrition. The modern high fat diet combined with physical inactivity has resulted in an international epidemic of obesity and overweight. According to the World Health Organization, there were last year for the first time in human history as many obese as starving people in the world.

The extensive data linking overweight and obesity to adverse health outcomes are well established and incontrovertible. ${ }^{4}$ The health consequences of obesity include some of the most common chronic diseases in the world. Obesity is an independent risk factor for heart disease, the most common killer disease in

Table - Inter-relationship between depression and obesity.

1. Depression and obesity frequently co-exist.

2. Both disorders are substantial health problems in worldwide.

3. Depressed mood can be a side effect of obesity treatments.

4. Weight gain and obesity can be a side effect of antidepressant treatments

5. Several neuropeptidergic and neurotransmitter systems, involving molecules as $\mathrm{CRH}$, NPY, serotonin, and norepinephrine, are involved in the regulation of mood as well as body weight.

6. Depression and obesity are important risk factors for cardiovascular disease, potentially resulting or worsening the metabolic syndrome.

7. Genetic polymorphisms may underlie the predisposition both to cardiovascular disease and to depression.

8. Drugs used in our depression study predominantly affect either serotonin or norepinephrine in the CNS.

9. Obesity treatment includes central inhibition of both serotonin and norepinephrine reuptake. developed countries. Non-insulin dependent diabetes mellitus, hypertension and stroke, hyperlipidemia, osteoarthritis, and sleep apnea are all more common in obese individuals. Weight loss of only 20 pounds can be associated with marked reductions in the risk of these chronic diseases. Conversely, adult weight gain is associated with increased risk of breast cancer in postmenopausal women.

Obesity is a heterogeneous disease that can be caused by genetic, environmental, social, economic, endocrine, metabolic and psychiatric causes. There are very few cases of obesity that are caused by specific genetic defects. In most cases there are partially inherited individual differences in metabolism and energy efficiency, which underlie body weight regulation. Therefore, while genetics determines the potential for obesity - diet, exercise, and lifestyle determine whether and to what extent obesity develops. There is an epidemic of obesity among children. The Surgeon General of the United States has made amelioration of childhood obesity a major health goal for the year 2010.

Most psychiatric disorders are no more common in obese individual, but the incidence of depression and anxiety is increased in obese compared to non-obese individuals. More research is needed on the observed association of obesity and depression. Weight gain and obesity can be an important adverse reaction to psychotropic drugs, including antidepressants and antipsychotics, both of which are used in the treatment of affective disorders.

There are many genes that are involved in energy metabolism and obesity. These genes include among others those coding for molecules related to the following neurotransmitters, neuropeptides, their receptors, synthesizing enzymes, and second messenger signaling systems: serotonin, norepinephrine, dopamine, leptin, NPY (neuropeptide Y), MC4 (melanocortin4 receptor), POMC (pro-opiomelanocortin), $\mathrm{CRH}$ (corticotropin releasing hormone), urocortin, $\mathrm{MCH}$ (melanin-concetrating hormone), galanin, leptin, adiponectin, ghrelin, CART, orexins, CCK (cholecytokinin), bombesin, GH (growth hormone), GLP1 (glucagon-like peptide-1), agouti-related, peptides, UCP2/ UCP3 (uncoupling protein 2 and 3), beta-3 adrenergic receptor. Several of these molecules are also thought to be abnormally regulated in depression.

In addition to the possibility of shared pathophysiologic mecha- 
nisms, obesity and depression have shared consequences, such as cardiovascular morbidity. Both disorders can contribute to the metabolic syndrome, which is the term used for the combination of obesity, high blood pressure, hyperlipidemia, and glucose intolerance, resulting in high risk for heart disease. Depression can by itself cause increase in food intake and body weight (changes in body weight are part of the official diagnostic criteria of depression). Moreover, depression can cause hypercortisolemia, which may enhance glucose intolerance, blood pressure, and adiposity. Depression also tends to be associated with decreased exercise rates. Therefore, major depression can by itself lead to the metabolic syndrome. Furthermore, recent data suggest that the same genetic factors, such as polymorphisms in the angiotensin I converting enzyme (ACE) gene, may underlie the predisposition to major depression and to heart disease. ${ }^{5,6}$ It is unknown whether the overlap of depression and obesity are syngergistic in terms of deterioration of cardiovascular function. The hypothesis that the overlap of obesity and depression is particularly deleterious to the cardiovascular system should therefore be tested by controlled studies.

In summary, obesity and depression are both major public health problems. These two disorders often overlap at multiple levels. Some of the brain circuits implicated in obesity are also thought to be dysregulated in depression. The same genetic factors might contribute to the predisposition both to depression and to heart disease. Moreover, drugs used to treat obesity act predominantly on the same neurotransmitter systems (such as norepinephrine and serotonin) as the drugs used in the treatment of depression. Mood changes can be a side effect of obesity treatments and weight gain can be a side effect of antidepressant treatments. Finally, both disorders can result in the metabolic syndrome, potentially increasing cardiovascular and metabolic morbidity. Increased emphasis is needed on the study of the behavioral mechanisms underlying obesity and on the metabolic and cardiovascular consequences of depression. We recommend that the study of these two disorders be brought together. Behavioral scientists working in depression can contribute to elucidate the behavioral basis of obesity and endocrinologists with expertise in obesity can use their skills to dissect the metabolic and cardiovascular consequences of depression. The integration of basic and clinical investigation in the fields of depression and obesity has the potential to bring new perspectives that will advance research and benefit patients.

\section{Julio Licinio Ma-Li Wong}

Laboratory for Pharmacogenomics Interdepartmental Clinical Pharmacology Center Neuropsychiatric Institute David Geffen School of Medicine at UCLA Los Angeles, USA

\section{References}

1. Bray G, Brouchard C, James W. Definitions and proposed current classification of obesity. Int J Obes Relat Metab Disord 1995;19(Suppl 4):S41-4.

2. Elmquist JK, Maratos-Flier E, Saper CB, Flier JS. Unraveling the central nervous system pathways underlying responses to leptin. Nat Neurosci 1998; 1:445-50.

3. Schwartz MW, Woods SC, Seeley RJ, Barsh GS, Baskin DG, Leibel RL. Is the energy homeostasis system inherently biased toward weight gain? Diabetes 2003;52:232-8.

4. National task force on the prevention and treatment of obesity. Overweight, obesity, and health risk. Arch Intern Med 2000;160:898-904.

5. Bondy B, Baghai TC, Zill P, Bottlender R, Jaeger M, Minov C, et al. Combined action of the ACE D- and the G-protein beta3 T-allele in major depression: a possible link to cardiovascular disease? Mol Psychiatry 2002;7:1120-6.

6. Licinio J, Yildiz B, Wong M-L. Depression and cardiovascular disease: co-occurrence or shared genetic substrates? Mol Psychiatry 2002;7:1031-2. 